\title{
Impact of diabetes mellitus on the prognostic value of the neutrophil-lymphocyte ratio in renal cell carcinoma
}

\author{
YANGQIN ZHENG $^{1^{*}}$, LIAN MIN BAO $^{2 *}$, JUNJIE YE $^{3^{*}}$, YUE PAN $^{4}$, QINQUAN WANG $^{4}$ and XIAOMIN GAO ${ }^{5}$ \\ ${ }^{1}$ Department of Hematology, The Third Clinical Institute Affiliated to Wenzhou Medical University, \\ People's Hospital of Wenzhou, Wenzhou, Zhejiang 325006; ${ }^{2}$ Department of Respiratory Medicine, Ruian People's Hospital, \\ The Third Affiliated Hospital of The Wenzhou Medical University, Wenzhou, Zhejiang 325200; ${ }^{3}$ Department of Urology, \\ Sixth Affiliated Hospital of Wenzhou Medical University, Lishui People's Hospital, Lishui, Zhejiang 323000; \\ ${ }^{4}$ Department of Urology, The First Affiliated Hospital of Wenzhou Medical University, Wenzhou, Zhejiang 325006; \\ ${ }^{5}$ Department of Urology, Changhai Hospital, Second Military Medical University, Shanghai 200433, P.R. China
}

Received August 7, 2018; Accepted November 7, 2018

DOI: $10.3892 /$ etm.2018.7093

\begin{abstract}
The aim of the present study was to evaluate the effect of diabetes mellitus (DM) on the neutrophil-lymphocyte ratio (NLR)-based prediction of the prognosis of patients with renal cell carcinoma (RCC). The data of 662 patients who had undergone nephrectomy for RCC between January 2004 and July 2014 were retrospectively reviewed. X-tile analysis was used to determine the optimal cutoff value for the NLR. Kaplan-Meier curves were drawn and the log-rank test was applied to determine the impact of the NLR (high vs. low) on the overall survival (OS) and metastasis-free survival (MFS). Univariate and multivariate Cox regression analyses were used to identify prognostic factors for OS and MFS. The median follow-up period after surgery was 50.35 months (range, 30.30-85.08 months). The optimal cutoff value of the NLR was determined to be 3.2 using X-tile software. In the analysis of total subjects, patients with a high NLR $(\geq 3.2)$ had significantly worse OS and MFS rates than those with a low NLR $(<3.2)(21.60 \%$ vs. $78.40 \%, \mathrm{P}=0.001$ for OS and $21.60 \%$ vs. $78.40 \%, \mathrm{P}<0.0001$ for MFS). In the non-DM subgroup, the OS and MFS rates of patients with a high NLR were significantly worse compared with those of patients with a low NLR ( $21.69 \%$ vs. $78.31 \%, \mathrm{P}=0.003$ for $\mathrm{OS}$ and $21.69 \%$ vs. $78.31 \%$,
\end{abstract}

Correspondence to: Dr Xiaomin Gao, Department of Urology, Changhai Hospital, Second Military Medical University, 168 Changhai Road, Yangpu, Shanghai 200433, P.R. China

E-mail: doctorgaoxiaomin@163.com

*Contributed equally

Abbreviations: RCC, renal cell carcinoma; NLR, neutrophil-lymphocyte ratio; DM, diabetes mellitus; OS, overall survival; MFS, metastasis-free survival; CSS, cancer-specific survival; SSIGN, Stage, Size, Grade and Necrosis Score

Key words: renal cell carcinoma, neutrophil-lymphocyte ratio, diabetes mellitus, prognosis
$\mathrm{P}<0.001$ for MFS). In the DM subgroup, although a high NLR was still associated with the MFS (NLR $\geq 3.2,21.43 \%$ vs. $\mathrm{NLR}<3.2,78.57 \%$; $\mathrm{P}=0.015)$, it was no longer associated with the OS $(\mathrm{NLR} \geq 3.2,21.43 \%$ vs. $\mathrm{NLR}<3.2,78.57 \%$; $\mathrm{P}=0.192)$. Furthermore, multivariate analysis identified the NLR as a risk factor for OS and MFS in all patients [hazard ratio $(\mathrm{HR})=1.77$, 95\% confidence interval $(\mathrm{CI})$ : 1.04-3.01, $\mathrm{P}=0.037$; and $\mathrm{HR}=2.31,95 \% \mathrm{CI}: 1.45-3.70, \mathrm{P}<0.001$, respectively) and in the non-DM subgroup (HR=2.03, 95\% CI: $1.05-3.93, \mathrm{P}=0.036$; and $\mathrm{HR}=2.57,95 \% \mathrm{CI}: 1.47-4.49, \mathrm{P}=0.001$, respectively), but not in the DM subgroup $(\mathrm{P}>0.05)$. In conclusion, $\mathrm{DM}$ is a factor that impairs the evaluation of the prognosis of RCC using NLR.

\section{Introduction}

The neutrophil-lymphocyte ratio (NLR) is a novel indicator of sub-clinical inflammation and has been identified as an independent risk factor for postoperative outcome in patients with various types of cancer (1). Previous studies have demonstrated that an elevated NLR is a significant adverse prognostic factor regarding overall survival (OS), disease-free survival and progression-free survival of metastatic or non-metastatic cancer patients (1). For liver cancer, Xue et al (2) performed a meta-analysis of 26 studies comprising 4,461 patients, which indicated that a high NLR was independently associated with poor OS and disease-free survival. For colorectal cancer, Walsh et al (3) reported that the pre-operative NLR is an independent risk factor and a predictor of worse OS and cancer-specific survival (CSS). The prognostic value of the NLR has also been confirmed in other cancer types, including small-cell lung (4), gastric (5) and ovarian cancer (6).

Previous studies have demonstrated that the preoperative NLR is a predictor of post-operative outcomes in patients with either non-metastatic $(7,8)$ or metastatic renal cell carcinoma (RCC) $(9,10)$. To date, several scoring systems and nomograms have been developed to improve the accuracy of survival prediction for localized RCC, including the Stage, Size, Grade, and Necrosis Score (SSIGN) (11), the Leibovich score as a modified version of the SSIGN score (12), the University of the California Los Angeles Integrated Staging System (13) 
and Karakiewicz's nomogram (14). These predictive models include the pathological tumor-nodes-metastasis (pTNM) stage and clinical criteria (Eastern Cooperative Oncology Group or Karnofsky), histological grading criteria (Fuhrman), imaging parameters (e.g. tumor size) and biological criteria (e.g. hemoglobin, neutrophils). However, the NLR has not been included in any of the abovementioned prognostic scoring systems for RCC. As a sensitive indicator of the inflammatory status, the NLR may be influenced by various clinical factors. Several studies have confirmed the significant association between the NLR and diabetes mellitus (DM) $(15,16)$. In addition, previous meta-analyses have indicated that DM increases the risk of the occurrence of $\operatorname{RCC}(17,18)$; furthermore, the increasing incidence of pre-existing DM may increase the incidence of RCC. The aim of the present study was to explore whether DM affects the NLR-based evaluation of the prognosis of patients with RCC after surgery.

\section{Patients and methods}

Patients. The present study retrospectively reviewed 662 consecutive patients with non-metastatic RCC treated with nephrectomy between January 2004 and July 2014 at the Department of Urology of the First Affiliated Hospital of Wenzhou Medical University, Wenzhou, China). Clinical data, including clinicopathologic and hematologic records, were collected and retrospectively analyzed.

Follow-up. Patients were generally followed up every 3-6 months for the first 2 years and annually thereafter by performing blood and urine tests, cystoscopy and image examination. Information on patient death was obtained from outpatient medical records, telephone interviews or the patient's social security death index. The OS was defined as the interval from the time-point of surgery to the date of death from all causes and metastasis-free survival (MFS) was defined as the interval from surgery to the date of recurrence of radiologically or histologically confirmed distant metastasis, according to the treating physician's assessment and radiologic criteria. The primary endpoint of the present study was MFS.

Statistical analysis. The NLR was calculated as the neutrophil count divided by the lymphocyte count. X-tile software (version 3.6.1; Yale University, New Haven CT, USA) was applied to calculate the discriminatory ability of NLR to identify the optimal cutoff value. The association of the clinicopathologic characteristics with low and high NLR was assessed using either Fisher's exact test or the Student's t-test and Pearson's Chi-square test. Kaplan-Meier survival curves were drawn to estimate OS and MFS and significant differences were determined using the log-rank test. Univariate analysis was performed with using Cox logistic regression. Variables with $\mathrm{P}<0.05$ in the univariate analysis were included in the subsequent multivariate analysis. Multivariate analysis was performed using Cox regression analysis. All tests were two-sided and $\mathrm{P}<0.05$ was considered to indicate a statistically significant difference. Statistical analyses were performed using the SPSS software package version 22.0 (IBM Corp., Armonk, NY, USA).

\section{Results}

Clinicopathological characteristics. A total of 662 consecutive patients were included in the current study, with $243(36.71 \%)$ females and $419(63.29 \%)$ males. The mean age of the cohort was 61.70 (12.65) years. To determine the most suitable cutoff value for NLR, X-tile software was applied with the MFS as the endpoint, and a cutoff value of NLR=3.2 was obtained (Fig. 1). The $\chi^{2} \log$-rank value of NLR was 21.15. Therefore, patients were divided into two groups according to the cutoff value: NLR $<3.2$ and $N L R \geq 3.2$. The patient population comprised 519 patients $(78.40 \%)$ with a low NLR and $143(21.60 \%)$ with a high NLR. A total of 662 patients with non-metastatic RCC, with a mean follow-up duration of 59.21 months (median, 50.35 months; range, 30.30-85.08 months) were included in the present study. During the follow-up, 74 patients $(11.18 \%)$ experienced distant metastasis and $60(9.06 \%)$ died, of which 41 cases $(6.19 \%)$ were cancer-specific deaths. The baseline clinicopathologic characteristics are summarized in Table I. Patients with a high NLR were more likely to be older, and had higher neutrophil and lower lymphocyte counts, as well as an advanced pathologic $\mathrm{T}$ stage and tumor grade (all $\mathrm{P}<0.05)$. The two groups were comparable with regard to sex, American Society of Anesthesiologists (ASA) grade, body mass index (BMI), type of surgery, mean tumor size, histologic subtype, platelet count and history of DM.

Factors affecting OS in total subjects. Univariate and multivariate analyses were used to determine the predictive value of the NLR value regarding the clinical prognosis of patients with RCC. According to the univariate analysis, an NLR of $\geq 3.2$, a higher age ( $\geq 65$ years), a higher ASA grade ( $\geq$ III), a higher BMI $\left(\geq 25 \mathrm{~kg} / \mathrm{m}^{2}\right)$, a larger mean tumor size $(\geq 7 \mathrm{~cm})$, a higher pathological $\mathrm{T}$ stage $(\geq 3)$ and a higher Fuhrman grade $(\geq 3)$ were significantly associated with poorer OS (all $\mathrm{P}<0.05$; Table II and Fig. 2A). Multivariate analysis revealed that an NLR of $\geq 3.2$, a higher age ( $\geq 65$ years), a higher BMI $\left(\geq 25 \mathrm{~kg} / \mathrm{m}^{2}\right)$, a larger mean tumor size $(\geq 7 \mathrm{~cm})$, a higher pathological T stage $(\geq 3)$ and higher a Fuhrman grade $(\geq 3)$ were independent negative prognostic factors regarding OS.

Factors affecting MFS in total subjects. According to the univariate analysis, an NLR of $\geq 3.2$, a higher age ( $\geq 65$ years), a higher ASA grade ( $\geq$ III), a higher BMI $\left(\geq 25 \mathrm{~kg} / \mathrm{m}^{2}\right)$, a larger mean tumor size $(\geq 7 \mathrm{~cm})$, a higher pathological $\mathrm{T}$ stage $(\geq 3)$ and a higher Fuhrman grade $(\geq 3)$ were significantly associated with poor MFS (All $\mathrm{P}<0.05$; Table III and Fig. 3A). Multivariate analysis revealed that an NLR of $\geq 3.2$, a higher age ( $\geq 65$ years), a higher BMI $\left(\geq 25 \mathrm{~kg} / \mathrm{m}^{2}\right)$, a larger mean tumor size $(\geq 7 \mathrm{~cm})$, a higher pathological $\mathrm{T}$ stage $(\geq 3)$ and a higher Fuhrman grade $(\geq 3)$ were independent prognostic factors associated with poor MFS.

Subgroup analysis. The patients were then divided into two subgroups according to the absence or presence of DM. There was no significant difference in NLR values between the two groups as indicated by the t-test $(\mathrm{P}=0.654$; Fig. 4). Furthermore, according to univariate analysis, DM did not significantly affect OS or MFS ( $\mathrm{P}=0.712$ or 0.536 , respectively), so that no subsequent multivariate analysis was performed for this factor. 
Table I. Patient characteristics and influence of the NLR.

\begin{tabular}{|c|c|c|c|c|}
\hline Factor & Total $(n=662)$ & $\operatorname{NLR}<3.2(\mathrm{n}=519)$ & $\operatorname{NLR} \geq 3.2(n=143)$ & P-value \\
\hline Age (years) & $61.70 \pm 12.65$ & $61.10 \pm 12.42$ & $63.90 \pm 13.27$ & 0.019 \\
\hline Sex & & & & 0.203 \\
\hline Female & $243(36.71)$ & $197(37.96)$ & $46(32.17)$ & \\
\hline Male & $419(63.29)$ & $322(62.04)$ & $97(67.83)$ & \\
\hline ASA grade & & & & 0.110 \\
\hline $\mathrm{I}$ & 85 (12.84) & $72(13.87)$ & $13(9.09)$ & \\
\hline II & $532(80.36)$ & $416(80.16)$ & $116(81.12)$ & \\
\hline III & $45(6.80)$ & $31(5.97)$ & $14(9.79)$ & \\
\hline BMI $\left(\mathrm{kg} / \mathrm{m}^{2}\right)$ & $23.16 \pm 3.04$ & $23.26 \pm 3.12$ & $22.82 \pm 2.71$ & 0.130 \\
\hline Type of surgery & & & & 0.262 \\
\hline Partial nephrectomy & $143(21.60)$ & $117(22.54)$ & $26(18.18)$ & \\
\hline Radical nephrectomy & $519(78.40)$ & $402(77.46)$ & $117(81.82)$ & \\
\hline Mean tumor size (SD; cm) & $4.91(3.42)$ & $4.85(3.46)$ & $5.14(3.30)$ & 0.375 \\
\hline Pathological T stage & & & & 0.046 \\
\hline pT1 & $514(77.64)$ & $415(79.96)$ & $99(69.23)$ & \\
\hline pT2 & $77(11.63)$ & $56(10.79)$ & $21(14.69)$ & \\
\hline pT3 & $62(9.37)$ & $42(8.09)$ & $20(13.98)$ & \\
\hline pT4 & $9(1.36)$ & $6(1.16)$ & $3(2.10)$ & \\
\hline Fuhrman grade & & & & 0.005 \\
\hline 1 & $210(31.72)$ & $173(33.33)$ & $37(25.87)$ & \\
\hline 2 & $282(42.60)$ & $229(44.12)$ & $53(37.06)$ & \\
\hline 3 & $148(22.36)$ & $101(19.46)$ & $47(32.87)$ & \\
\hline 4 & $22(3.32)$ & $16(3.09)$ & $6(4.20)$ & \\
\hline Histologic subtype & & & & 0.682 \\
\hline Clear cell carcinoma & $581(87.76)$ & $454(87.48)$ & $127(88.81)$ & \\
\hline Papillary carcinoma & $41(6.20)$ & $31(5.97)$ & $10(6.99)$ & \\
\hline Chromophobe carcinoma & $36(5.44)$ & $31(5.97)$ & $5(3.50)$ & \\
\hline Collecting duct carcinoma & $1(0.15)$ & $1(0.19)$ & $0(0.00)$ & \\
\hline Unclassified carcinoma & $3(0.45)$ & $2(0.39)$ & $1(0.70)$ & \\
\hline Mean nutrophil count $\times 10^{9}$ (mean; SD) & $4.12 \pm 1.68$ & $3.63 \pm 1.15$ & $5.88 \pm 2.08$ & $<0.001$ \\
\hline Lymphocyte count x $10^{9}$ (mean; SD) & $1.78 \pm 0.62$ & $1.93 \pm 0.58$ & $1.22 \pm 0.39$ & $<0.001$ \\
\hline Platelet count (mean; SD) & $216.46 \pm 70.66$ & $213.97 \pm 68.01$ & $225.48 \pm 79.13$ & 0.085 \\
\hline Diabetes mellitus & & & & 0.938 \\
\hline No & $438(66.16)$ & $343(66.09)$ & $95(66.43)$ & \\
\hline Yes & $224(33.84)$ & $176(33.91)$ & $48(33.57)$ & \\
\hline
\end{tabular}

Values are expressed as $\mathrm{n}(\%)$, the mean \pm standard deviation or mean (SD). The normal range of neutrophil count, lymphocyte count and platelet count were 1.80-6.30x10 $/ 1,1.10-3.20 \times 10^{9} / 1$ and 125-350x $10^{9} / 1$, respectively. BMI, body mass index; NLR, neutrophil-lymphocyte ratio; ASA, American Society of Anesthesiologists; SD, standard deviation.

Kaplan-Meier analysis indicated that in the non-DM group, a high pre-operative NLR ( $\geq 3.2)$ was significantly associated with a worse OS and MFS (Fig. 2C and 3C). While a high NLR was still associated with MFS in the DM group, it was no longer associated with OS (Fig. 2C and 3C). Uni- and multivariate analysis of factors affecting survival was then performed for the DM and non-DM subgroups individually, as summarized in Tables II and III. In the non-DM group, the results demonstrated that a high NLR was still an independent predictive factor of poor OS and MFS in patients with
RCC. However, in the DM group, an NLR of $\geq 3.2$ was not a significant independent prognostic factor.

\section{Discussion}

According to previous studies, an elevated NLR is associated with poor prognosis for patients with RCC (7-10). In the present study, a retrospective review was performed to investigate the correlation between the pre-operative NLR and the post-surgical outcomes for patients with RCC. In line with the 

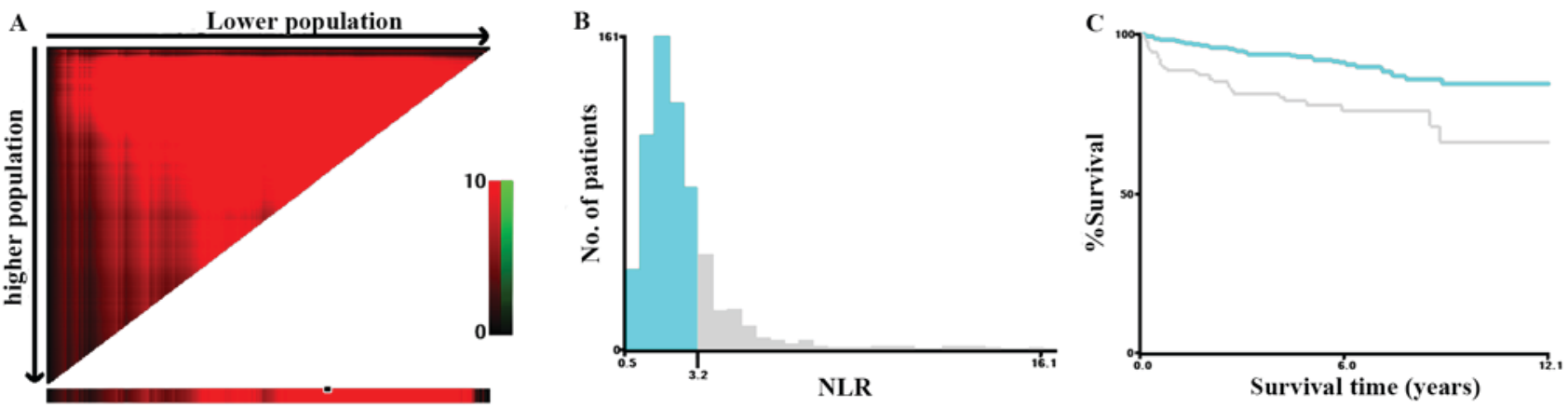

Figure 1. X-tile analyses of metastasis-free survival was performed to determine the optimal cutoff value for the NLR. NLR, neutrophil-lymphocyte ratio. (A) The red coloration of the cut-points indicates an inverse correlation with survival; while the green coloration represents direct associations. (B) Histograms of the entire cohort. (C) Kaplan-Meier plots. The optimal cut-off values highlighted by black circles in (A) are presented in of the entire cohort (B).
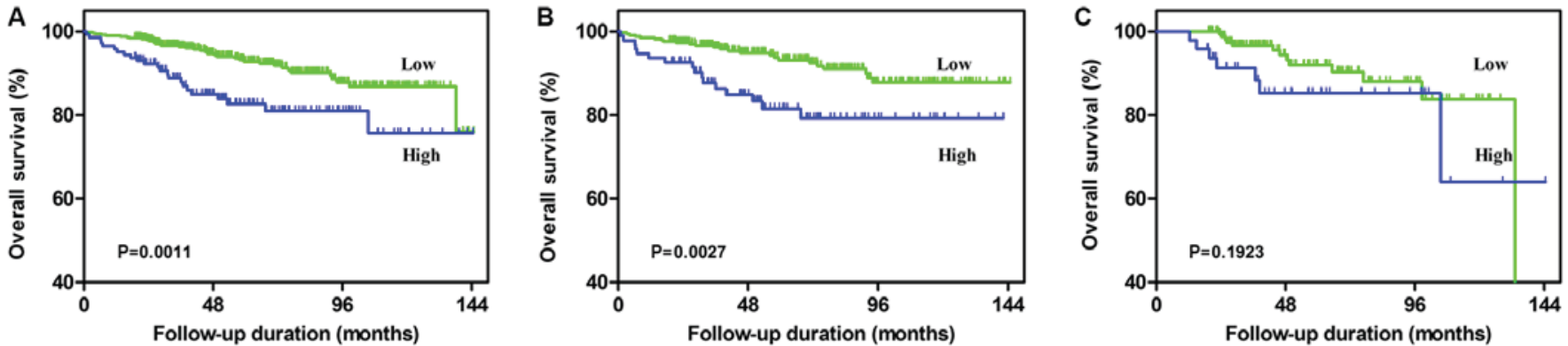

Figure 2. Kaplan-Meier curves for overall survival of patients stratifed by their neutrophil-lymphocyte ratio (high vs. low). (A) All patients, (B) patients without $\mathrm{DM}$ and (C) patients with DM. DM, diabetes mellitus.

results of previous studies (1,7-10), it was demonstrated that a high NLR has a prognostic value in patients with RCC. A high NLR was associated with old age, high neutrophil and lymphocyte counts, as well as an advanced pathologic $\mathrm{T}$ stage and tumor grade. In addition, multivariate analysis identified that an NLR of $\geq 3.2$ is an independent adverse prognostic factor for RCC patients. The present study therefore confirmed that NLR is a significant prognostic factor for RCC and may be useful for tailoring therapies for patients with RCC. However, unlike other systemic inflammatory indicators, including C-reactive protein (CRP) and the platelet-to-lymphocyte ratio, NLR has still not been incorporated in any clinical evaluation system for RCC. Therefore, the primary aim of the present study was to identify the possible reasons.

Various studies have emphasized the importance of inflammation in carcinogenesis (19-21). According to them, one potential mechanism is that the response of systemic inflammation to various physiological challenges is characterized by increased neutrophil and decreased lymphocyte counts, largely favoring tumor development by preventing or suppressing the activation of anti-tumor cells in the immune system. Of note, cancer cells themselves are able to recruit and activate various types of leukocytes, particularly neutrophils and monocytes (22). Therefore, an increased NLR is thought to provide a favorable microenvironment for tumor development and metastasis. The NLR has also been associated with poor prognosis in conditions other than cancer, including cardiovascular diseases $(23,24)$, respiratory diseases $(25)$ and hypertension (26). Therefore, NLR is a non-specific parameter, which may be affected by concurrent conditions, including chronic obstructive pulmonary disease and coronary chronic total occlusion.

Previous meta-analyses indicated that DM may increase the risk of RCC $(17,18)$. Of note, an increased NLR was also reported to be associated with DM, and a high NLR value may be a significant predictive marker of DM (15). In the present study, a high NLR was identified as an independent risk factor for RCC regarding OS and MFS. No significant difference in NLR values was identified between the patient groups with and without DM, and DM was not a significant influencing factor of OS and MFS according to the multivariate regression analysis. However, in the multivariate analysis for the subgroup of patients with DM, an elevated NLR was no longer identified as an independent predictive factor of OS and MFS, but it was still a significant independent predictor in the subgroup of patients without DM. The HR value of NLR for OS and MFS increased from 1.77 in the total subject group to 2.03 in the non-DM group. A further increase was identified from 2.31 in the total subject group to 2.57 in the non-DM group. Furthermore, the results of multivariate analysis demonstrate that an elevated NLR is no longer identified as the independent factor of OS and MFS in the DM group, but may still serve as an independent predictor in patients without DM. All of the above indicates that DM is a disturbance factor in the evaluation of prognosis of RCC using NLR. The link between DM and RCC-associated mortality has been evaluated in several previous studies. In 2013, Ha et al (27) published a multi-institutional analysis including a total of 2,597 patients, revealing that DM is an independent prognostic factor for recurrence-free survival 


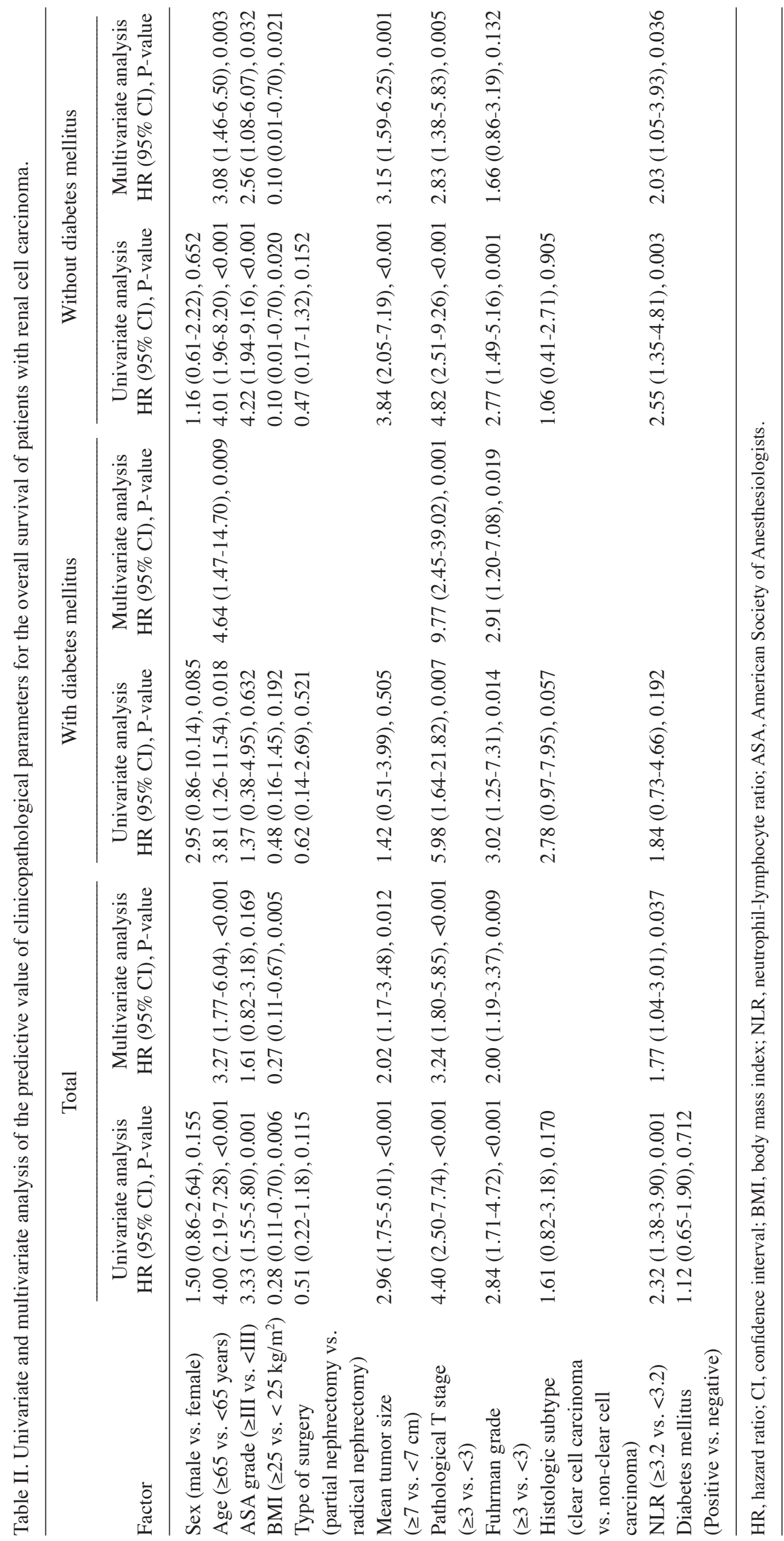




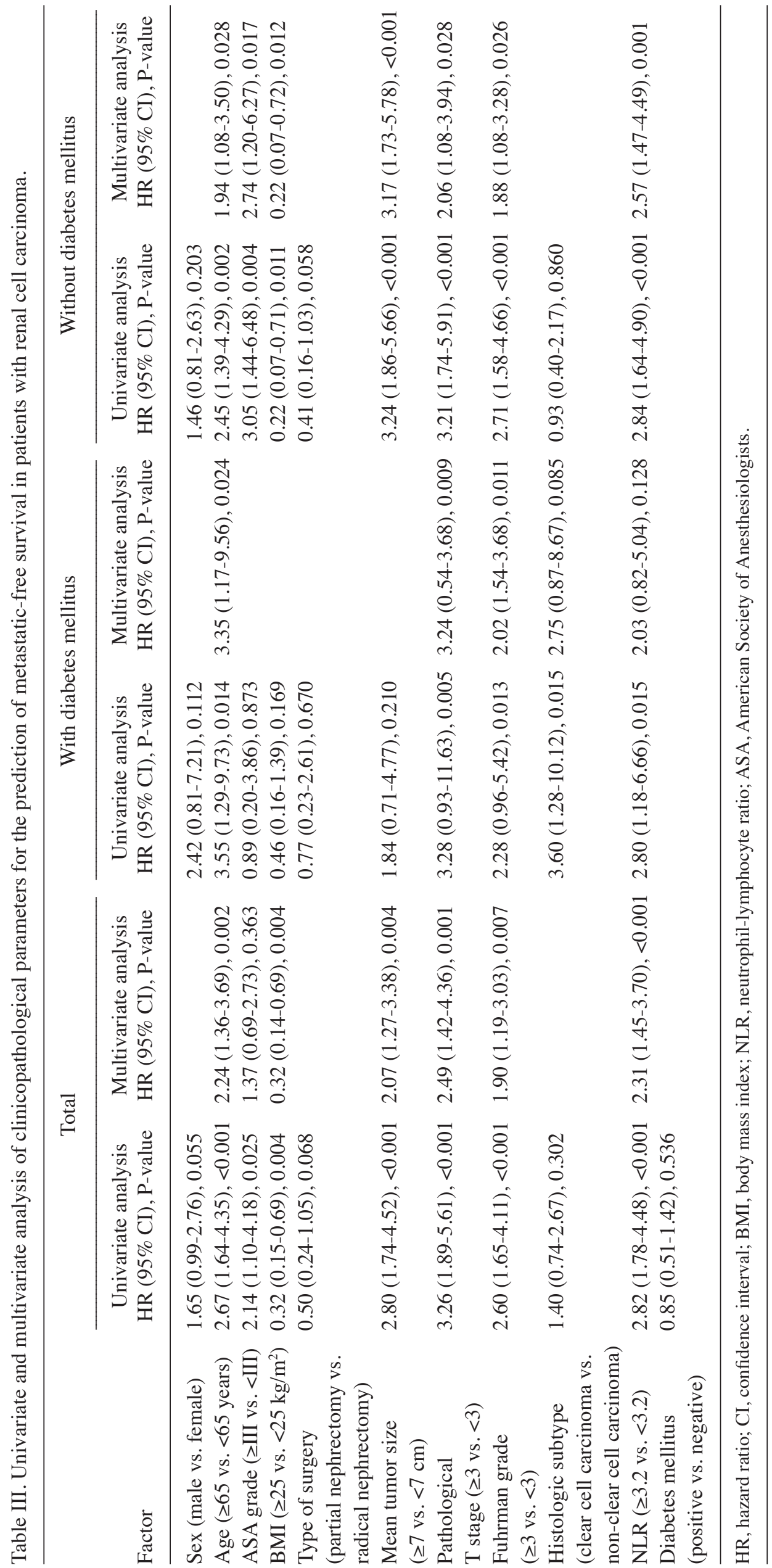



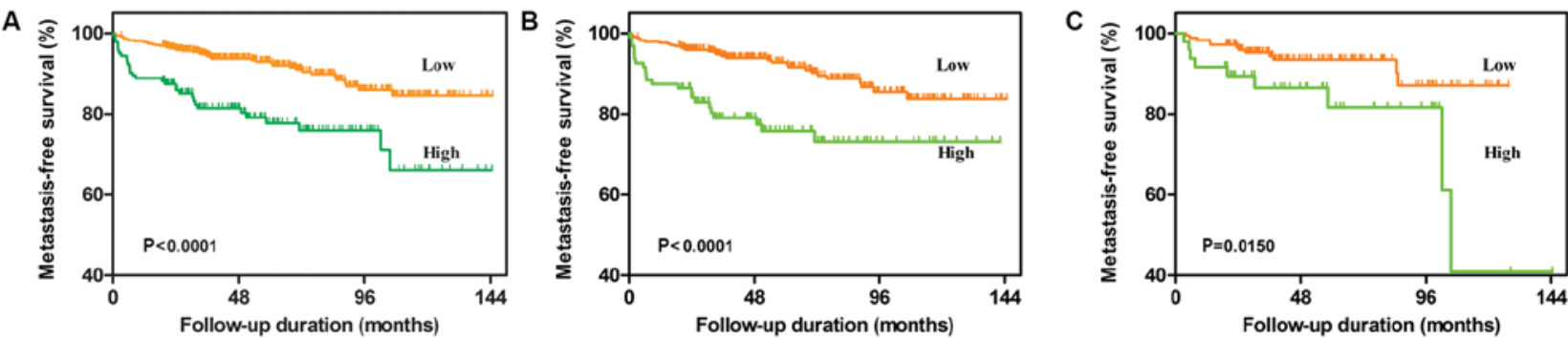

Figure 3. Kaplan-Meier curves for metastasis-free survival of patients stratified by their neutrophil-lymphocyte ratio (high vs. low). (A) All patients, (B) patients without DM and (C) patients with DM. DM, diabetes mellitus.

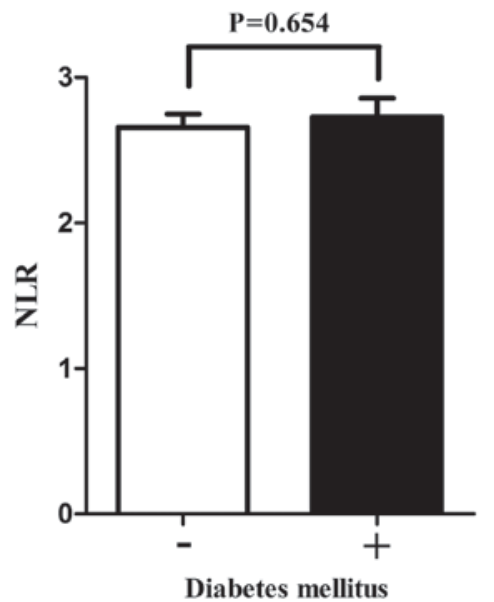

Figure 4. Comparison of NLR between renal cell carcinoma patients with or without diabetes mellitus. NLR, neutrophil-lymphocyte ratio.

(RFS), CSS and OS. Another meta-analysis published in 2015 including a total of 20,199 patients, also revealed a significant negative impact of DM on OS, CSS and RFS in patients with RCC (28). Therefore, more attention should be paid when evaluating the prognosis of patients with RCC based on the NLR and DM.

All of the factors included in the pTNM classification are able to provide reliable prognostic information. In addition, these factors, e.g. the pathological $\mathrm{T}$ stage, are confirmed from pathological specimens and are stable predictors that are not influenced by any physiological factors. However, the most widely used prognostic nomograms and risk scores for RCC, which are based on the pTNM stage, are established post-operatively. These conventional prognostic factors have limited accuracy. Furthermore, it is also important for clinical surgeons to identify prognostic factors prior to the surgery in order to conceive patient-specific therapeutic strategies. In recent years, the prognostic value of biomarkers of inflammation, including the NLR and CRP, has been evidenced in patients with cancer. Hu et al (7) reported that the NLR is superior to CRP as a predictor of RCC. However, the NLR is easily affected by numerous physiological factors, but its application still has potential value in patients with no underlying conditions (e.g. DM or cardiopulmonary diseases), as it is easily measured, inexpensive and repeatable.

Of note, the present study has several limitations. First, it was a retrospective single-center study. It may be argued that the size of the study population was insufficient; however, the results were representative and reliable, as our department is the largest urologic cancer center in the South of Zhejiang Province and provides access to a wide variety of patients with RCC Furthermore, the cutoff value of NLR was different in other studies (29). However, a threshold of NLR $=3$ is considered reasonable for RCC (29), which is close to the cutoff value of NLR=3.2 used in the present study. Finally, the measurement of NLR may be complicated by concurrent conditions, including infections and inflammation, as well as by certain medications. In the present study, all of the blood specimens were obtained prior to surgery. In addition, surgeons commonly delay procedures for patients with active infections. Therefore, it is unlikely that the NLR was influenced by any infections. However, the complicating effect of concurrent inflammatory conditions was not completely excluded.

In conclusion, the present study indicated that the prognostic value of NLR for patients with RCC was impaired by concurrent DM, as indicated by a subgroup analysis of patients with and without DM. It is therefore suggested that DM should be considered when evaluating the prognostic value of NLR in patients with RCC.

\section{Acknowledgements}

The authors thank Editage for English language editing.

\section{Funding}

No funding was received.

\section{Availability of data and materials}

The datasets used and/or analyzed during the present study are available from the corresponding author on reasonable request.

\section{Authors' contributions}

XG conceived and designed the study, $\mathrm{YZ}, \mathrm{BL}$, and $\mathrm{YJ}$ acquired the data, YP and QW analyzed and interpreted the data, and $\mathrm{XG}$ drafted the manuscript.

\section{Ethics approval and consent to participate}

The present study was approved by the ethics committee of the First Affiliated Hospital of Wenzhou Medical University (Wenzhou, China). The study protocol is in accordance with the Declaration of Helsinki. 


\section{Patient consent for publication}

Not applicable.

\section{Competing interests}

The authors declare that they have no competing interests.

\section{References}

1. Templeton AJ, McNamara MG, Šeruga B, Vera-Badillo FE, Aneja P, Ocaña A, Leibowitz-Amit R, Sonpavde G, Knox JJ, Tran B, et al: Prognostic role of neutrophil-to-lymphocyte ratio in solid tumors: A systematic review and meta-analysis. J Natl Cancer Inst 106: dju124, 2014.

2. Xue TC, Zhang L, Xie XY, Ge NL, Li LX, Zhang BH, Ye SL and Ren ZG: Prognostic significance of the neutrophil-to-lymphocyte ratio in primary liver cancer: A meta-analysis. PLoS One 9: e96072, 2014.

3. Walsh SR, Cook EJ, Goulder F, Justin TA and Keeling NJ: Neutrophil-lymphocyte ratio as a prognostic factor in colorectal cancer. J Surg Oncol 91: 181-184, 2005

4. Kang MH, Go SI, Song HN, Lee A, Kim SH, Kang JH, Jeong BK, Kang KM, Ling $\mathrm{H}$ and Lee GW: The prognostic impact of the neutrophil-to-lymphocyte ratio in patients with small-cell lung cancer. Br J Cancer 111: 452-460, 2014.

5. Lieto E, Galizia G, Auricchio A, Cardella F, Mabilia A, Basile N, Del Sorbo G, Castellano P, Romano C, Orditura M and Napolitano V: Preoperative neutrophil to lymphocyte ratio and lymphocyte to monocyte ratio are prognostic factors in gastric cancers undergoing surgery. J Gastrointest Surg 21: 1764-1774, 2017.

6. Cho H, Hur HW, Kim SW, Kim SH, Kim JH, Kim YT and Lee K: Pre-treatment neutrophil to lymphocyte ratio is elevated in epithelial ovarian cancer and predicts survival after treatment. Cancer Immunol Immunother 58: 15-23, 2009.

7. Hu H, Yao X, Xie X, Wu X, Zheng C, Xia W and Ma S: Prognostic value of preoperative NLR, dNLR, PLR and CRP in surgical renal cell carcinoma patients. World J Urol 35: 261-270, 2017.

8. Ohno Y, Nakashima J, Ohori M, Hatano T and Tachibana M: Pretreatment neutrophil-to-lymphocyte ratio as an independent predictor of recurrence in patients with nonmetastatic renal cell carcinoma. J Urol 184: 873-878, 2010.

9. Cetin B, Berk V, Kaplan MA, Afsar B, Tufan G, Ozkan M, Isikdogan A, Benekli M, Coskun U and Buyukberber S: Is the pretreatment neutrophil to lymphocyte ratio an important prognostic parameter in patients with metastatic renal cell carcinoma? Clin Genitourin Cancer 11: 141-148, 2013.

10. Fox P, Hudson M, Brown C, Lord S, Gebski V, De Souza P and Lee CK: Markers of systemic inflammation predict survival in patients with advanced renal cell cancer. Br J Cancer 109: 147-153, 2013.

11. Frank I, Blute ML, Cheville JC, Lohse CM, Weaver AL and Zincke H: An outcome prediction model for patients with clear cell renal cell carcinoma treated with radical nephrectomy based on tumor stage, size, grade and necrosis: The SSIGN score. J Urol 168: 2395-2400, 2002.

12. Leibovich BC, Blute ML, Cheville JC, Lohse CM, Frank I, Kwon ED, Weaver AL, Parker AS and Zincke H: Prediction of progression after radical nephrectomy for patients with clear cell renal cell carcinoma: A stratification tool for prospective clinical trials. Cancer 97: 1663-1671, 2003.

13. Patard JJ, Kim HL, Lam JS, Dorey FJ, Pantuck AJ, Zisman A, Ficarra V, Han KR, Cindolo L, De La Taille A, et al: Use of the University of California Los Angeles integrated staging system to predict survival in renal cell carcinoma: An international multicenter study. J Clin Oncol 22: 3316-3322, 2004.

14. Karakiewicz PI, Suardi N, Capitanio U, Jeldres C, Ficarra V, Cindolo L, de la Taille A, Tostain J, Mulders PF, Bensalah K, et al: A preoperative prognostic model for patients treated with nephrectomy for renal cell carcinoma. Eur Urol 55: 287-295, 2009.

15. Lou M, Luo P, Tang R, Peng Y, Yu S, Huang W and He L: Relationship between neutrophil-lymphocyte ratio and insulin resistance in newly diagnosed type 2 diabetes mellitus patients. BMC Endocr Disord 15: 9, 2015.
16. Shiny A, Bibin YS, Shanthirani CS, Regin BS, Anjana RM, Balasubramanyam M, Jebarani S and Mohan V: Association of neutrophil-lymphocyte ratio with glucose intolerance: An indicator of systemic inflammation in patients with type 2 diabetes. Diabetes Technol Ther 16: 524-530, 2014

17. Larsson SC and Wolk A: Diabetes mellitus and incidence of kidney cancer: A meta-analysis of cohort studies. Diabetologia 54: 1013-1018, 2011

18. Bao C, Yang X, Xu W, Luo H, Xu Z, Su C and Qi X: Diabetes mellitus and incidence and mortality of kidney cancer: A meta-analysis. J Diabetes Complications 27: 357-364, 2013.

19. Balkwill FR and Mantovani A: Cancer-related inflammation: Common themes and therapeutic opportunities. Semin Cancer Biol 22: 33-40, 2012.

20. Chan AT, Ogino S and Fuchs CS: Aspirin and the risk of colorectal cancer in relation to the expression of COX-2. N Eng J Med 356: 2131-2142, 2007.

21. Sparmann A and Bar-Sagi D: Ras-induced interleukin-8 expression plays a critical role in tumor growth and angiogenesis. Cancer Cell 6: 447-458, 2004.

22. Stoppacciaro A, Melani C, Parenza M, Mastracchio A, Bassi C, Baroni C, Parmiani G and Colombo MP: Regression of an established tumor genetically modified to release granulocyte colony-stimulating factor requires granulocyte-T cell cooperation and T cell-produced interferon gamma. J Exp Med 178: 151-161, 1993.

23. Avci A, Elnur A, Göksel A, Serdar F, Servet I, Atilla K, Mustafa TM, Cuneyt T, Yeliz G, Mustafa B and Metin EA: The relationship between neutrophil/lymphocyte ratio and calcific aortic stenosis. Echocardiography 31: 1031-1035, 2014

24. Balta S, Demirkol S, Kucuk U, Celik T, Ozturk C and Iyisoy A: The relationship between neutrophil-lymphocyte ratio and coronary collateral circulation. Perfusion 29: 367-368, 2014

25. Yao C, Liu X and Tang Z: Prognostic role of neutrophil-lymphocyte ratio and platelet-lymphocyte ratio for hospital mortality in patients with AECOPD. Int J Chron Obstruct Pulmon Dis 12: 2285-2290, 2017.

26. Demir M: The relationship between neutrophil lymphocyte ratio and non-dipper hypertension. Clin Exp Hypertens 35: 570-573, 2013.

27. Ha YS, Kim WT, Yun SJ, Lee SC, Kim WJ, Park YH, Kang SH, Hong SH, Byun SS and Kim YJ: Multi-institutional analysis of localized renal cell carcinoma that demonstrates the impact of diabetic status on prognosis after nephrectomy. Ann Surg Oncol 20: 3662-3668, 2013 .

28. Chen L, Li H, Gu L, Ma X, Li X, Gao Y, Zhang Y, Shen D, Fan Y, Wang B, et al: The impact of diabetes mellitus on renal cell carcinoma prognosis: A meta-analysis of cohort studies. Medicine (Baltimore) 94: e1055, 2015.

29. Boissier R, Campagna J, Branger N, Karsenty G and Lechevallier E: The prognostic value of the neutrophil-lymphocyte ratio in renal oncology: A review. Urol Oncol 35: 135-141, 2017. 